\title{
Multi-point boundary value problems for a class of Riemann-Liouville fractional differential equations
}

\author{
Bingxian $\mathrm{Li}^{1}$, Shurong Sun ${ }^{*}$, Yanan $\mathrm{Li}^{1}$ and Ping Zhao ${ }^{2}$
}

\author{
"Correspondence: \\ sshrong@163.com \\ ischool of Mathematical Sciences, \\ University of Jinan, Jinan, Shandong \\ 250022, P.R. China \\ Full list of author information is \\ available at the end of the article
}

\begin{abstract}
In this paper, we shall study the existence and uniqueness of solutions for the multi-point boundary value problem of fractional differential equations $D_{0^{+}}^{\alpha} u(t)+f(t, u(t))=0,0<t<1,2<\alpha \leq 3$, with boundary conditions $u(0)=0$, $D_{0^{+}}^{\beta} u(0)=0, D_{0^{+}}^{\beta} u(1)=\sum_{i=1}^{m-2} b_{i} D_{0^{+}}^{\beta} u\left(\xi_{i}\right), 1 \leq \beta \leq 2$, involving Riemann-Liouville fractional derivatives $D_{0^{+}}^{\alpha}$ and $D_{0^{+}}^{\beta}$. We use the nonlinear alternative of Leray-Schauder and the Banach contraction mapping principle to obtain the existence and uniqueness of solutions. Some examples are given to show the applicability of our main results.
\end{abstract}

MSC: $34 \mathrm{~A} 08 ; 34 \mathrm{~K} 10$

Keywords: fractional differential equations; multi-point boundary value problem; existence and uniqueness; fixed point theorem

\section{Introduction}

Fractional calculus is the study and application of arbitrary order differential and integral theory; see [1-5]. It is consistent with integer order calculus and a natural extension of the integer order calculus. Fractional differential equations are developed accompanied by fractional calculus. In recent years, with the wide applications of fractional calculus in the fields of physical, mechanical, biological, ecological, engineering, etc., the theory of fractional calculus has been paid more and more attention. Especially the study of fractional differential equations as abstracted from practical problems attracts much attention of many mathematicians.

Boundary value problems for fractional differential equations belong to the important issues for the theory of fractional differential equations. A lot of papers focused on two-point boundary value problems of fractional ordinary differential equations [6-16], boundary value problems of fractional difference equations $[17,18]$, and problems of fractional functional differential equations [19-25].

However, the results dealing with multi-point boundary value problems of fractional differential equations are relatively scarce [26-31].

In 2010, Li et al. [26] considered the existence and uniqueness for nonlinear fractional differential equation of the type

$$
D_{0^{+}}^{\alpha} u(t)+f(t, u(t))=0, \quad 0<t<1,1<\alpha \leq 2,
$$


where $D_{0^{+}}^{\alpha}$ is the standard Riemann-Liouville fractional order derivative, subject to the boundary conditions

$$
u(0)=0, \quad D_{0^{+}}^{\beta} u(1)=a D_{0^{+}}^{\beta} u(\xi), \quad 0 \leq \beta \leq 1 .
$$

They obtained the existence and multiplicity results of positive solutions by using some fixed point theorems.

In 2011, Yang et al. [27] discussed the existence and uniqueness for a multi-point boundary value problem of the fractional differential equation

$$
\begin{aligned}
& D_{0^{+}}^{\alpha} u(t)+f(t, u(t))=0, \quad 0<t<1,1<\alpha \leq 2, \\
& u(0)=0, \quad D_{0^{+}}^{\beta} u(1)=\sum_{i=1}^{m-2} b_{i} D_{0^{+}}^{\beta} u\left(\xi_{i}\right), \quad 0 \leq \beta \leq 1,
\end{aligned}
$$

where $D_{0^{+}}^{\alpha}$ and $D_{0^{+}}^{\beta}$ are the Riemann-Liouville fractional derivatives. By fixed point theorem, they obtained the existence and uniqueness results.

In the previous related studies, scholars mostly used a fixed point theorem in cones and the Schauder fixed point theorem to solve some classes of boundary value problems. On the other hand, the study of these classes of problems has been only limited to the low order.

Motivated by their excellent results and the methods, in this paper, we investigate the existence and uniqueness for the multi-point fractional differential equation

$$
\begin{aligned}
& D_{0^{+}}^{\alpha} u(t)+f(t, u(t))=0, \quad 0<t<1, \\
& u(0)=0, \quad D_{0^{+}}^{\beta} u(0)=0, \quad D_{0^{+}}^{\beta} u(1)=\sum_{i=1}^{m-2} b_{i} D_{0^{+}}^{\beta} u\left(\xi_{i}\right),
\end{aligned}
$$

where $D_{0^{+}}^{\alpha}$ and $D_{0^{+}}^{\beta}$ are the Riemann-Liouville fractional derivatives, $2<\alpha \leq 3,1 \leq \beta \leq 2$ with $\alpha \geq \beta+1, m \in \mathbb{N}^{+}$with $m>2,0<\xi_{1}<\xi_{2}<\cdots<\xi_{m-2}<1$.

To the best of our knowledge, no one has studied the existence of positive solutions for boundary value problems (1.1) and (1.2). Our main results of this paper are in extending the results in [27] from low order to high order case. Our problem allows the boundary condition to depend on the lower fractional derivative $D_{0^{+}}^{\beta}$, which leads to extra difficulties. In particular, the condition $D_{0+}^{\beta} u(0)=0$ involves not only the properties of the function $u(t)$ at zero but also the slope of tangent which pass through zero if $\beta=1$. Key tools in finding our main results are the nonlinear alternative of the Leray-Schauder and the Banach contraction mapping principle.

The plan of this paper is as follows. In Section 2, we shall give some definitions and lemmas to prove our main results. In Section 3, we establish the existence and uniqueness of solutions to multi-point boundary value problems (1.1) and (1.2) by the Banach contraction mapping principle, and we investigate the existence of solutions for (1.1) and (1.2) by the nonlinear alternative of Leray-Schauder. In Section 4, examples are presented to illustrate the main results.

In order to facilitate our study, we make the following assumptions: 
$\left(\mathrm{H}_{1}\right) f:[0,1] \times[0, \infty) \rightarrow[0, \infty)$ is a continuous function;

$\left(\mathrm{H}_{2}\right) b_{i} \geq 0(i=1,2, \ldots, m-2), \sum_{i=1}^{m-2} b_{i}^{2} \neq 0$, and $A=\sum_{i=1}^{m-2} b_{i} \xi_{i}^{\alpha-\beta-1}<1$.

\section{Preliminaries}

For the convenience of the reader, we present here some necessary definitions and lemmas from the fractional calculus theory.

Definition 2.1 ([4] $)$ The fractional integral of order $\alpha(\alpha>0)$ of a function $f:(0,+\infty) \rightarrow \mathbb{R}$ is given by

$$
I_{0^{+}}^{\alpha} f(t)=\frac{1}{\Gamma(\alpha)} \int_{0}^{t} \frac{f(s)}{(t-s)^{1-\alpha}} d s,
$$

where $\Gamma(\cdot)$ is the gamma function, provided that the right side is point-wise defined on $(0,+\infty)$.

Definition 2.2 ([4]) The Riemann-Liouville fractional derivative of order $\alpha>0$ of a continuous function $f:(0,+\infty) \rightarrow \mathbb{R}$ is given by

$$
D_{0^{+}}^{\alpha} f(t)=\frac{1}{\Gamma(n-\alpha)}\left(\frac{d}{d t}\right)^{n} \int_{0}^{t}(t-s)^{n-\alpha-1} f(s) d s
$$

where $\Gamma(\cdot)$ is the gamma function, provided that the right side is point-wise defined on $(0,+\infty)$ and $n=[\alpha]+1,[\alpha]$ stands for the largest integer less than $\alpha$.

Lemma 2.1 ([4]) Let $\alpha>-1, \beta>0$ and $t>0$. Then

$$
D_{0^{+}}^{\beta} t^{\alpha}=\frac{\Gamma(\alpha+1)}{\Gamma(\alpha-\beta+1)} t^{\alpha-\beta}
$$

Lemma 2.2 ([4]) Assume that $u(t) \in J(0,1) \cap L(0,1), D_{0^{+}}^{\alpha} u \in J(0,1) \cap L(0,1)$ with the Riemann-Liouville fractional derivative of order $\alpha>0$, then

$$
I_{0^{+}}^{\alpha} D_{0^{+}}^{\alpha} u(t)=u(t)+c_{1} t^{\alpha-1}+c_{2} t^{\alpha-2}+\cdots+c_{N} t^{\alpha-N},
$$

where $c_{i} \in R, i=1,2, \ldots, N$, and $N$ is the smallest integer greater than or equal to $\alpha$.

Lemma 2.3 For Riemann-Liouville fractional derivatives, we have

$$
D_{0^{+}}^{\beta} \int_{0}^{t}(t-s)^{\alpha-1} f(s, u(s)) d s=\frac{\Gamma(\alpha)}{\Gamma(\alpha-\beta)} \int_{0}^{t}(t-s)^{\alpha-\beta-1} f(s, u(s)) d s,
$$

where $f \in C[0,1], \alpha, \beta$ are two constants with $\alpha>\beta \geq 0$.

Proof From

$$
D_{0^{+}}^{\alpha} I_{0^{+}}^{\alpha} f(t)=f(t), \quad I_{0^{+}}^{\alpha} I_{0^{+}}^{\beta} f(t)=I_{0^{+}}^{\alpha+\beta} f(t),
$$


we get

$$
\begin{aligned}
D_{0^{+}}^{\beta} \int_{0}^{t}(t-s)^{\alpha-1} f(s, u(s)) d s & =D_{0^{+}}^{\beta} \Gamma(\alpha) \frac{1}{\Gamma(\alpha)} \int_{0}^{t}(t-s)^{\alpha-1} f(s, u(s)) d s \\
& =D_{0^{+}}^{\beta} \Gamma(\alpha) I_{0^{+}}^{\alpha} f(t, u(t))=\Gamma(\alpha) D_{0^{+}}^{\beta} I_{0^{+}}^{\alpha} f(t, u(t)) \\
& =\Gamma(\alpha) D_{0^{+}}^{\beta} I_{0^{+}}^{\beta} I_{0^{+}}^{\alpha-\beta} f(t, u(t))=\Gamma(\alpha) I_{0^{+}}^{\alpha-\beta} f(t, u(t)) \\
& =\Gamma(\alpha) \frac{1}{\Gamma(\alpha-\beta)} \int_{0}^{t}(t-s)^{\alpha-\beta-1} f(s, u(s)) d s .
\end{aligned}
$$

Then we obtain the result. The proof is complete.

The following lemma is fundamental in proofs of our main results.

Lemma 2.4 ([32]) Let $E$ be a Banach space with $C \subseteq E$ closed and convex. Assume $U$ is a relatively open subset of $C$ with $0 \in U$ and $T: \bar{U} \rightarrow E$ is a completely continuous operator, $T(\bar{U})$ is bounded. Then either

(c) Thas a fixed point in $\bar{U}$; or

$\left(c_{2}\right)$ there exist $a \in \in \partial U$ and $\lambda \in(0,1)$ with $u=\lambda T u$.

\section{Main results}

For convenience, assume $E=C[0,1]$ is a Banach space with the maximum norm $\|x\|=$ $\max _{0 \leq t \leq 1}|x(t)|$ for $x \in C[0,1]$. Let $r>0$ and define $X_{r}=\{u: u \in C[0,1],\|u\|<r\}$, which is the subset of $C[0,1]$. Let $M=\max \{|f(t, u)|:(t, u) \in[0,1] \times[-r, r]\}$.

Lemma 3.1 Let $\left(\mathrm{H}_{1}\right)$ and $\left(\mathrm{H}_{2}\right)$ hold. Then the boundary value problem of the following fractional differential equation:

$$
\begin{aligned}
& D_{0^{+}}^{\alpha} u(t)+f(t, u(t))=0, \quad 0<t<1,2<\alpha \leq 3, \\
& u(0)=0, \quad D_{0^{+}}^{\beta} u(0)=0, \quad D_{0^{+}}^{\beta} u(1)=\sum_{i=1}^{m-2} b_{i} D_{0^{+}}^{\beta} u\left(\xi_{i}\right), \quad 1 \leq \beta \leq 2,
\end{aligned}
$$

has a unique solution:

$$
\begin{aligned}
u(t)= & -\int_{0}^{t} \frac{1}{\Gamma(\alpha)}(t-s)^{\alpha-1} f(s, u(s)) d s+\frac{1}{(1-A) \Gamma(\alpha)} \int_{0}^{1}(1-s)^{\alpha-\beta-1} t^{\alpha-1} f(s, u(s)) d s \\
& -\frac{1}{(1-A) \Gamma(\alpha)} \sum_{i=1}^{m-2} b_{i} \int_{0}^{\xi_{i}}\left(\xi_{i}-s\right)^{\alpha-\beta-1} t^{\alpha-1} f(s, u(s)) d s .
\end{aligned}
$$

Proof By Definition 2.1 and Lemma 2.2, we get

$$
u(t)=-\int_{0}^{t} \frac{1}{\Gamma(\alpha)}(t-s)^{\alpha-1} f(s, u(s)) d s+c_{1} t^{\alpha-1}+c_{2} t^{\alpha-2}+c_{3} t^{\alpha-3}
$$

is the general solution of equation (1.1). By boundary condition $u(0)=0$, we find that

$$
c_{3}=0 .
$$


In view of Lemma 2.3 and $D_{0^{+}}^{\beta} u(0)=0$, we have

$$
\begin{aligned}
D_{0^{+}}^{\beta} u(t)= & D_{0^{+}}^{\beta}\left(-\int_{0}^{t} \frac{1}{\Gamma(\alpha)}(t-s)^{\alpha-1} f(s, u(s)) d s\right)+c_{1} \frac{\Gamma(\alpha)}{\Gamma(\alpha-\beta)} t^{\alpha-\beta-1} \\
& +c_{2} \frac{\Gamma(\alpha-1)}{\Gamma(\alpha-\beta-1)} t^{\alpha-\beta-2} .
\end{aligned}
$$

For $\alpha \in(2,3], \beta \in[1,2]$ and $\alpha \geq \beta+1$, we have $\alpha-\beta-2 \in[-1,0]$. Thus $c_{2}=0$. By

$$
D_{0^{+}}^{\beta} u(1)=\sum_{i=1}^{m-2} b_{i} D_{0^{+}}^{\beta} u\left(\xi_{i}\right),
$$

we get

$$
\begin{aligned}
c_{1}= & \frac{1}{(1-A) \Gamma(\alpha)} \int_{0}^{1}(1-s)^{\alpha-\beta-1} f(s, u(s)) d s \\
& -\frac{1}{(1-A) \Gamma(\alpha)} \sum_{i=1}^{m-2} b_{i} \int_{0}^{\xi_{i}}\left(\xi_{i}-s\right)^{\alpha-\beta-1} f(s, u(s)) d s .
\end{aligned}
$$

Then the boundary value problem has a unique solution

$$
\begin{aligned}
u(t)= & -\int_{0}^{t} \frac{1}{\Gamma(\alpha)}(t-s)^{\alpha-1} f(s, u(s)) d s+\frac{1}{(1-A) \Gamma(\alpha)} \int_{0}^{1}(1-s)^{\alpha-\beta-1} t^{\alpha-1} f(s, u(s)) d s \\
& -\frac{1}{(1-A) \Gamma(\alpha)} \sum_{i=1}^{m-2} b_{i} \int_{0}^{\xi_{i}}\left(\xi_{i}-s\right)^{\alpha-\beta-1} t^{\alpha-1} f(s, u(s)) d s .
\end{aligned}
$$

The proof is completed.

Set $f(t, u(t))=g(t)$ in Lemma 3.1. Since $f:[0,1] \times[0,+\infty) \rightarrow[0,+\infty)$ is a continuous function, we deduce that function $u$ is a solution of the boundary value problem (1.1) and (1.2) if and only if it satisfies

$$
\begin{aligned}
u(t)= & -\int_{0}^{t} \frac{1}{\Gamma(\alpha)}(t-s)^{\alpha-1} f(s, u(s)) d s+\frac{1}{(1-A) \Gamma(\alpha)} \int_{0}^{1}(1-s)^{\alpha-\beta-1} t^{\alpha-1} f(s, u(s)) d s \\
& -\frac{1}{(1-A) \Gamma(\alpha)} \sum_{i=1}^{m-2} b_{i} \int_{0}^{\xi_{i}}\left(\xi_{i}-s\right)^{\alpha-\beta-1} t^{\alpha-1} f(s, u(s)) d s
\end{aligned}
$$

Let $T: \bar{X}_{r} \rightarrow E$ be the operator defined by

$$
\begin{aligned}
T u(t)= & -\int_{0}^{t} \frac{1}{\Gamma(\alpha)}(t-s)^{\alpha-1} f(s, u(s)) d s \\
& +\frac{1}{(1-A) \Gamma(\alpha)} \int_{0}^{1}(1-s)^{\alpha-\beta-1} t^{\alpha-1} f(s, u(s)) d s \\
& -\frac{1}{(1-A) \Gamma(\alpha)} \sum_{i=1}^{m-2} b_{i} \int_{0}^{\xi_{i}}\left(\xi_{i}-s\right)^{\alpha-\beta-1} t^{\alpha-1} f(s, u(s)) d s
\end{aligned}
$$


Lemma 3.2 $T: \bar{X}_{r} \rightarrow E$ is a completely continuous operator.

Proof For $f(t, u)$ continuous, it is easy to see that $T: \bar{X}_{r} \rightarrow E$ is continuous.

For $2<\alpha \leq 3,1 \leq \beta \leq 2$ and $\bar{X}_{r}$ is bounded, then for any $u \in \bar{X}_{r}$ and $t \in[0,1]$,

$$
\begin{aligned}
|T u(t)| \leq & \mid-\int_{0}^{t} \frac{1}{\Gamma(\alpha)}(t-s)^{\alpha-1} f(s, u(s)) d s \\
& +\frac{1}{(1-A) \Gamma(\alpha)} \int_{0}^{1}(1-s)^{\alpha-\beta-1} t^{\alpha-1} f(s, u(s)) d s \\
& -\frac{1}{(1-A) \Gamma(\alpha)} \sum_{i=1}^{m-2} b_{i} \int_{0}^{\xi_{i}}\left(\xi_{i}-s\right)^{\alpha-\beta-1} t^{\alpha-1} f(s, u(s)) d s \mid \\
\leq & \frac{M}{\Gamma(\alpha)} \int_{0}^{t}(t-s)^{\alpha-1} d s+\frac{M t^{\alpha-1}}{(1-A) \Gamma(\alpha)} \int_{0}^{1}(1-s)^{\alpha-\beta-1} d s \\
& +\frac{M t^{\alpha-1}}{(1-A) \Gamma(\alpha)} \sum_{i=1}^{m-2} b_{i} \int_{0}^{\xi_{i}}\left(\xi_{i}-s\right)^{\alpha-\beta-1} d s \\
\leq & \frac{M}{(1-A) \Gamma(\alpha)}\left(\frac{1-A}{\alpha}+\frac{1}{\alpha-\beta}+\frac{1}{\alpha-\beta} \sum_{i=1}^{m-2} b_{i} \xi_{i}^{\alpha-\beta}\right) .
\end{aligned}
$$

Thus

$$
\|T u\| \leq \frac{M}{(1-A) \Gamma(\alpha)}\left(\frac{1-A}{\alpha}+\frac{1}{\alpha-\beta}+\frac{1}{\alpha-\beta} \sum_{i=1}^{m-2} b_{i} \xi_{i}^{\alpha-\beta}\right) \quad \text { for all } u \in \bar{X}_{r} .
$$

Hence $\left\{T u, u \in \bar{X}_{r}\right\}$ is bounded.

On the other hand, we will show that for any given $\varepsilon>0$, there exists

$$
\delta=\min \left\{1,\left(\frac{\varepsilon \Gamma(\alpha+1)(1-A)(\alpha-\beta)}{M\left((1-A)(\alpha-\beta)+\alpha+\alpha \sum_{i=1}^{m-2} b_{i} \xi_{i}^{\alpha-\beta}\right) 2^{\alpha}}\right)^{\frac{1}{\alpha-1}}\right\}
$$

for any $u \in \bar{X}_{r}, t_{1}, t_{2} \in[0,1]$, with $0<t_{2}-t_{1}<\delta$, we get

$$
\left|T u\left(t_{2}\right)-T u\left(t_{1}\right)\right|<\varepsilon \text {. }
$$

Thus $T: \bar{X}_{r}$ is completely continuous.

In fact, for any $u \in \bar{X}_{r}, t_{1}, t_{2} \in[0,1]$ with $t_{1}<t_{2}$, we have

$$
\begin{aligned}
\left|T u\left(t_{2}\right)-T u\left(t_{1}\right)\right| \\
\leq\left|\int_{0}^{t_{2}} \frac{1}{\Gamma(\alpha)}\left(t_{2}-s\right)^{\alpha-1} f(s, u(s)) d s-\int_{0}^{t_{1}} \frac{1}{\Gamma(\alpha)}\left(t_{1}-s\right)^{\alpha-1} f(s, u(s)) d s\right| \\
\quad+\mid \frac{t_{2}^{\alpha-1}}{(1-A) \Gamma(\alpha)} \int_{0}^{1}(1-s)^{\alpha-\beta-1} f(s, u(s)) d s \\
\quad-\frac{t_{1}^{\alpha-1}}{(1-A) \Gamma(\alpha)} \int_{0}^{1}(1-s)^{\alpha-\beta-1} f(s, u(s)) d s \mid
\end{aligned}
$$




$$
\begin{aligned}
& +\mid \frac{t_{2}^{\alpha-1}}{(1-A) \Gamma(\alpha)} \sum_{i=1}^{m-2} b_{i} \int_{0}^{\xi_{i}}\left(\xi_{i}-s\right)^{\alpha-\beta-1} f(s, u(s)) d s \\
& -\frac{t_{1}^{\alpha-1}}{(1-A) \Gamma(\alpha)} \sum_{i=1}^{m-2} b_{i} \int_{0}^{\xi_{i}}\left(\xi_{i}-s\right)^{\alpha-\beta-1} f(s, u(s)) d s \mid \\
\leq & M\left|\int_{0}^{t_{1}} \frac{\left(t_{2}-s\right)^{\alpha-1}-\left(t_{1}-s\right)^{\alpha-1}}{\Gamma(\alpha)} d s+\int_{t_{1}}^{t_{2}} \frac{\left(t_{2}-s\right)^{\alpha-1}}{\Gamma(\alpha)} d s\right| \\
& +\frac{M\left|t_{2}^{\alpha-1}-t_{1}^{\alpha-1}\right|}{(1-A) \Gamma(\alpha)} \int_{0}^{1}(1-s)^{\alpha-\beta-1} d s \\
& +\frac{M \sum_{i=1}^{m-2} b_{i}\left|t_{2}^{\alpha-1}-t_{1}^{\alpha-1}\right|}{(1-A) \Gamma(\alpha)} \int_{0}^{\xi_{i}}\left(\xi_{i}-s\right)^{\alpha-\beta-1} d s \\
= & \frac{M\left(t_{2}^{\alpha}-t_{1}^{\alpha}\right)}{\Gamma(\alpha+1)}+\frac{M\left|t_{2}^{\alpha-1}-t_{1}^{\alpha-1}\right|}{(1-A) \Gamma(\alpha)}\left(\frac{1}{\alpha-\beta}+\frac{1}{\alpha-\beta} \sum_{i=1}^{m-2} b_{i} \xi_{i}^{\alpha-\beta}\right) \\
= & \frac{M\left(t_{2}^{\alpha}-t_{1}^{\alpha}\right)}{\Gamma(\alpha+1)}+\frac{M\left(1+\sum_{i=1}^{m-2} b_{i} \xi_{i}^{\alpha-\beta}\right)}{(\alpha-\beta)(1-A) \Gamma(\alpha)}\left|t_{2}^{\alpha-1}-t_{1}^{\alpha-1}\right| .
\end{aligned}
$$

(1) If $\delta \leq t_{1}<t_{2}<1$, by the mean value theorem, we have

$$
t_{2}^{\alpha}-t_{1}^{\alpha} \leq \alpha\left(t_{2}-t_{1}\right), \quad t_{2}^{\alpha-1}-t_{1}^{\alpha-1} \leq(\alpha-1)\left(t_{2}-t_{1}\right) \leq \alpha\left(t_{2}-t_{1}\right)
$$

(2) If $0 \leq t_{1}<\delta, t_{2}<2 \delta$, then

$$
t_{2}^{\alpha}-t_{1}^{\alpha} \leq t_{2}^{\alpha}<(2 \delta)^{\alpha}, \quad t_{2}^{\alpha-1}-t_{1}^{\alpha-1} \leq t_{2}^{\alpha-1}<(2 \delta)^{\alpha-1} .
$$

So

$$
\max \left\{t_{2}^{\alpha-1}-t_{1}^{\alpha-1}, t_{2}^{\alpha}-t_{1}^{\alpha}\right\} \leq 2^{\alpha} \delta^{\alpha-1}
$$

Thus

$$
\left|T u\left(t_{2}\right)-T u\left(t_{1}\right)\right|<\frac{M\left((1-A)(\alpha-\beta)+\alpha+\alpha \sum_{i=1}^{m-2} b_{i} \xi_{i}^{\alpha-\beta}\right) 2^{\alpha}}{\Gamma(\alpha+1)(1-A)(\alpha-\beta)} \delta^{\alpha-1}<\varepsilon .
$$

By the Arzela-Ascoli theorem, we conclude that $T: \bar{X}_{r} \rightarrow E$ is a completely continuous operator.

Theorem 3.1 Assume that there exists a constant $k>0$ such that

$$
|f(t, u)-f(t, v)| \leq k|u-v|
$$

where $u, v \in \mathbb{R}$. Then the boundary value problem (1.1)-(1.2) has a unique solution on $[0,1]$, if

$$
\frac{k\left((1-A)(\alpha-\beta)+\alpha+\alpha \sum_{i=1}^{m-2} b_{i} \xi_{i}^{\alpha-\beta}\right)}{(1-A)(\alpha-\beta) \Gamma(\alpha+1)}<1
$$

is satisfied. 
Proof By the definition of $T$, we have

$$
\begin{aligned}
|(T u)(t)-(T v)(t)| \leq & \frac{1}{\Gamma(\alpha)} \int_{0}^{t}(t-s)^{\alpha-1}|f(s, u(s))-f(s, v(s))| d s \\
& +\frac{1}{(1-A) \Gamma(\alpha)} \int_{0}^{1}(1-s)^{\alpha-\beta-1} t^{\alpha-1}|f(s, u(s))-f(s, v(s))| d s \\
& +\frac{1}{(1-A) \Gamma(\alpha)} \sum_{i=1}^{m-2} b_{i} \int_{0}^{\xi_{i}}\left(\xi_{i}-s\right)^{\alpha-\beta-1} t^{\alpha-1}|f(s, u(s))-f(s, v(s))| d s \\
\leq & k\|u-v\|\left(\frac{1}{\Gamma(\alpha)} \int_{0}^{t}(t-s)^{\alpha-1} d s+\frac{1}{(1-A) \Gamma(\alpha)} \int_{0}^{1}(1-s)^{\alpha-\beta-1} d s\right. \\
& \left.+\frac{1}{(1-A) \Gamma(\alpha)} \sum_{i=1}^{m-2} b_{i} \int_{0}^{\xi_{i}}\left(\xi_{i}-s\right)^{\alpha-\beta-1} d s\right) \\
= & \frac{k\left[(1-A)(\alpha-\beta)+\alpha+\alpha \sum_{i=1}^{m-2} b_{i} \xi_{i}^{\alpha-\beta}\right]}{(1-A)(\alpha-\beta) \Gamma(\alpha+1)}\|u-v\| .
\end{aligned}
$$

Hence, by the Banach contraction mapping principle, boundary value problems (1.1) and (1.2) have a unique solution on $[0,1]$. The proof is completed.

Now we study the existence of solutions for the boundary value problem of (1.1)-(1.2) by the nonlinear alternative of Leray-Schauder.

\section{Theorem 3.2 Suppose that the following condition are satisfied:}

$\left(\mathrm{a}_{1}\right)$ There exist a nonnegative function $g \in C[0,1]$ such that $g>0$ on the subset of $[0,1]$, and a nondecreasing function $h:[0, \infty) \rightarrow[0, \infty)$ such that $|f(t, u)| \leq g(t) h(\|u\|)$, where $(t, u) \in[0,1] \times \mathbb{R}$.

$\left(\mathrm{a}_{2}\right)$

$$
\sup _{r \in(0, \infty)} \frac{r}{g_{0} h(r)}>1,
$$

where

$$
\begin{aligned}
g_{0}= & \frac{1}{\Gamma(\alpha)} \int_{0}^{1}(1-s)^{\alpha-1} g(s) d s+\frac{1}{(1-A) \Gamma(\alpha)} \int_{0}^{1}(1-s)^{\alpha-\beta-1} g(s) d s \\
& +\frac{1}{(1-A) \Gamma(\alpha)} \sum_{i=1}^{m-2} b_{i} \int_{0}^{\xi_{i}}\left(\xi_{i}-s\right)^{\alpha-\beta-1} g(s) d s .
\end{aligned}
$$

Then boundary value problem (1.1)-(1.2) has at least one solution.

Proof In view of $\left(\mathrm{a}_{2}\right)$ and by the definition of supremum, we can choose a constant $r_{0} \in$ $(0, \infty)$ such that

$$
\frac{r_{0}}{g_{0} h\left(r_{0}\right)}>1 \text {. }
$$


By Lemma 3.2, we know that $T: \bar{X}_{r_{0}} \rightarrow E$ is completely continuous and $T\left(\bar{X}_{r_{0}}\right)$ is bounded. Suppose $\left(\mathrm{c}_{2}\right)$ in Lemma 2.4 holds, i.e. there exist a $\lambda \in(0,1), u \in \partial X_{r_{0}}$ such that

$$
u=\lambda T u .
$$

Then

$$
\begin{aligned}
u(t)= & \lambda\left(-\frac{1}{\Gamma(\alpha)} \int_{0}^{t}(t-s)^{\alpha-1} f(s, u(s)) d s+\frac{1}{(1-A) \Gamma(\alpha)} \int_{0}^{1}(1-s)^{\alpha-\beta-1} t^{\alpha-1} f(s, u(s)) d s\right. \\
& \left.-\frac{1}{(1-A) \Gamma(\alpha)} \sum_{i=1}^{m-2} b_{i} \int_{0}^{\xi_{i}}\left(\xi_{i}-s\right)^{\alpha-\beta-1} t^{\alpha-1} f(s, u(s)) d s\right) .
\end{aligned}
$$

In view of $\left(\mathrm{a}_{1}\right),\left(\mathrm{a}_{2}\right),(3.2)$, and $\|u\|=r_{0}$, we obtain

$$
\begin{aligned}
r_{0}= & \|u\| \leq \frac{h\left(r_{0}\right)}{\Gamma(\alpha)} \int_{0}^{1}(1-s)^{\alpha-1} g(s) d s+\frac{h\left(r_{0}\right)}{(1-A) \Gamma(\alpha)} \int_{0}^{1}(1-s)^{\alpha-\beta-1} g(s) d s \\
& +\frac{h\left(r_{0}\right)}{(1-A) \Gamma(\alpha)} \sum_{i=1}^{m-2} b_{i} \int_{0}^{\xi_{i}}\left(\xi_{i}-s\right)^{\alpha-\beta-1} g(s) d s \\
\leq & g_{0} h\left(r_{0}\right) .
\end{aligned}
$$

Hence we get $\frac{r_{0}}{g_{0} h\left(r_{0}\right)} \leq 1$, which is in contradiction with (3.1). Therefore, Lemma 2.4 guarantees that $T$ has at least a fixed point $u \in \bar{X}_{r_{0}}$. Then boundary value problem (1.1)-(1.2) has at least one solution. The proof is completed.

\section{Examples}

In this section, we will present some examples to illustrate our main results.

Example 4.1 Consider the following boundary value problem:

$$
\begin{aligned}
& D_{0^{+}}^{\frac{5}{2}} u(t)+\frac{u(t)}{10}+\sin ^{2} t+1=0, \quad 0<t<1, \\
& u(0)=0, \quad D_{0^{+}}^{\frac{3}{2}} u(0)=0, \quad D_{0^{+}}^{\frac{3}{2}} u(1)=\sum_{i=1}^{2} b_{i} D_{0^{+}}^{\frac{3}{2}} u\left(\xi_{i}\right),
\end{aligned}
$$

where $b_{1}=\frac{1}{10}, b_{2}=\frac{1}{5}, \xi_{1}=\frac{1}{10}, \xi_{2}=\frac{1}{5}$.

Here

$$
\alpha=\frac{5}{2}, \quad \beta=\frac{3}{2}, \quad f(t, u)=\frac{u}{10}+\sin ^{2} t+1, \quad \text { for }(t, u) \in[0,1] \times[0, \infty] .
$$

It is clear that $|f(t, u)-f(t, v)|=\frac{1}{10}|u-v|$ and

$$
\begin{aligned}
\frac{k\left((1-A)(\alpha-\beta)+\alpha+\alpha \sum_{i=1}^{m-2} b_{i} \xi_{i}^{\alpha-\beta}\right)}{(1-A)(\alpha-\beta) \Gamma(\alpha+1)} & =\frac{\frac{1}{10}\left(\left(1-\frac{3}{10}\right)\left(\frac{5}{2}-\frac{3}{2}\right)+\frac{5}{2}+\frac{5}{2} \times \frac{1}{20}\right)}{\left(1-\frac{3}{10}\right)\left(\frac{5}{2}-\frac{3}{2}\right) \Gamma\left(\frac{5}{2}\right) \times \frac{5}{2}} \\
& =\frac{91}{525 \sqrt{\pi}}<1 .
\end{aligned}
$$

By Theorem 3.1, we see that boundary value problem (4.1)-(4.2) has a unique solution. 
Example 4.2 Consider the following boundary value problem:

$$
\begin{aligned}
& D_{0^{+}}^{\frac{5}{2}} u(t)+t u^{2}(t) \sin u^{2}(t)=0, \quad 0<t<1, \\
& u(0)=0, \quad D_{0^{+}}^{\frac{3}{2}} u(0)=0, \quad D_{0^{+}}^{\frac{3}{2}} u(1)=\sum_{i=1}^{m-2} b_{i} D_{0^{+}}^{\frac{3}{2}} u\left(\xi_{i}\right),
\end{aligned}
$$

where $b_{1}=\frac{1}{10}, \xi_{1}=\frac{1}{10}$.

Here $\alpha=\frac{5}{2}, \beta=\frac{3}{2}$. Set $g(t)=t$ and $h(u)=u^{2}$. It is easy to see that

$$
|f(t, u)|=\left|t u^{2} \sin u^{2}\right| \leq t u^{2}, \quad \text { for }(t, u) \in[0,1] \times[0, \infty) .
$$

By simply calculating, we get

$$
\begin{aligned}
g_{0}= & \frac{1}{\Gamma(\alpha)} \int_{0}^{1}(1-s)^{\alpha-1} g(s) d s+\frac{1}{(1-A) \Gamma(\alpha)} \int_{0}^{1}(1-s)^{\alpha-\beta-1} g(s) d s \\
& +\frac{1}{(1-A) \Gamma(\alpha)} \sum_{i=1}^{m-2} b_{i} \int_{0}^{\xi_{i}}\left(\xi_{i}-s\right)^{\alpha-\beta-1} g(s) d s \\
= & \frac{7,405}{6,750 \sqrt{\pi}} \approx 0.61892 .
\end{aligned}
$$

Now

$$
\sup _{r \in(0, \infty)} \frac{r}{g_{0} h(r)}=\sup _{r \in(0, \infty)} \frac{r}{\frac{7,405 r^{2}}{6,750 \sqrt{\pi}}}=+\infty
$$

Hence by Theorem 3.2, we obtain the result that boundary value problem (4.3)-(4.4) has at least a solution.

\section{Competing interests}

The authors declare that they have no competing interests.

\section{Authors' contributions}

The authors declare that the study was realized in collaboration with the same responsibility. All authors read and approved the final manuscript.

\section{Author details}

${ }^{1}$ School of Mathematical Sciences, University of Jinan, Jinan, Shandong 250022, P.R. China. ${ }^{2}$ School of Electrical Engineering, University of Jinan, Jinan, Shandong 250022, P.R. China.

\section{Acknowledgements}

The authors sincerely thank the reviewers for their valuable suggestions and useful comments that have led to the present improved version of the original manuscript. This research is supported by the Natural Science Foundation of China (61374074), Natural Science Outstanding Youth Foundation of Shandong Province (JQ201119) and supported by Shandong Provincial Natural Science Foundation (ZR2012AM009, ZR2013AL003).

\section{Received: 30 September 2013 Accepted: 7 May 2014 Published: 20 May 2014}

\section{References}

1. Oldham, K, Spanier, J: The Fractional Calculus. Academic Press, New York (1974)

2. Miller, K, Ross, B: An Introduction to the Fractional Calculus and Fractional Differential Equation. Wiley, New York (1993)

3. Samko, S, Kilbas, A, Marichev, O: Fractional Integral and Derivative, Theory and Applications. Gordon \& Breach, Switzerland (1993) 
4. Podlubny, I: Fractional Differential Equations. Academic Press, San Diego (1999)

5. Kilbas, A, Srivastava, H, Trujillo, J: Theory and Applications of Fractional Differential Equations. Elsevier, Amsterdam (2006)

6. Agarwal, R, Benchohra, M, Hamani, A: Boundary value problems for fractional differential equations. Georgian Math. J. 16, 401-411 (2009)

7. Agarwal, $R, Z$, hou, $Y, H$, $Y$ : Existence of fractional neutral functional differential equations. Comput. Math. Appl. 59, 1095-1100 (2010)

8. Zhang, M, Sun, S, Zhao, Y, Yang, D: Existence of positive solutions for boundary value problems of fractional differential equations. J. Jinan Univ. 24, 205-208 (2010)

9. Zhao, Y, Sun, S, Han, Z, Li, Q: The existence of multiple positive solutions for boundary value problems of nonlinear fractional differential equations. Commun. Nonlinear Sci. Numer. Simul. 16(4), 2086-2097 (2011)

10. Zhao, Y, Sun, S, Han, Z, Li, Q: Positive solutions to boundary value problems of nonlinear fractional differential equations. Abstr. Appl. Anal. 2011, 390543 (2011)

11. Zhao, Y, Sun, S, Han, Z, Zhang, M: Positive solutions for boundary value problems of nonlinear fractional differential equations. Appl. Math. Comput. 217, 6950-6958 (2011)

12. Feng, W, Sun, S, Han, Z, Zhao, Y: Existence of solutions for a singular system of nonlinear fractional differential equations. Comput. Math. Appl. 62(3), 1370-1378 (2011)

13. Pan, Y, Han, Z: Existence of solutions for a coupled system of boundary value problem of nonlinear fractional differential equations. In: Proceedings of the 5th International Congress on Mathematical Biology, pp. 109-114. World Academic Press, Nanjing (2011)

14. Zhang, $X, L i u, L, W u, Y$ : Multiple positive solutions of a singular fractional differential equation with negatively perturbed term. Math. Comput. Model. 55, 1263-1274 (2012)

15. Zhang, S: Positive solutions for boundary-value problems of nonlinear fractional differential equations. Electron J. Differ. Equ. 36, 1072-6691 (2006)

16. Shi, A: Upper and lower solutions method and a fractional differential equation boundary value problem. Electron. J. Qual. Theory Differ. Equ. 30, 1-13 (2009)

17. Pan, Y, Han, Z, Sun, S, Zhao, Y: The existence of solutions to a system of discrete fractional boundary value problems. Abstr. Appl. Anal. 2012, 707631 (2012)

18. Pan, Y, Han, Z, Sun, S, Huang, Z: The existence and uniqueness of solutions to boundary value problems of fractional difference equations. Math. Sci. 6(7), 1-10 (2012)

19. Li, Y, Sun, S, Han, Z, Lu, H: The existence of positive solutions for boundary value problem of the fractional Sturm-Liouville functional differential equation. Abstr. Appl. Anal. 2013, 301560 (2013)

20. Zhou, Y, Tian, Y, He, Y: Floquet boundary value problems of fractional functional differential equations. Electron. J. Qual. Theory Differ. Equ. 50, 1-13 (2010)

21. Li, Y, Sun, S, Yang, D, Han, Z: Three-point boundary value problems of fractional functional differential equations with delay. Bound. Value Probl. 2013), 38 (2013)

22. Li, X, Song, L, Wei, J: Positive solutions for boundary value problem of nonlinear fractional functional differential equations. Appl. Math. Comput. 217, 9278-9285 (2011)

23. Bai, C: Existence of positive solutions for a functional fractional boundary value problem. Abstr. Appl. Anal. 2010, $127363(2010)$

24. Ouyang, Z, Chen, Y, Zou, S: Existence of positive solutions to a boundary value problem for a delayed nonlinear fractional differential system. Bound. Value Probl. 2011, 475126 (2011)

25. Zhao, Y, Chen, H, Huang, L: Existence of positive solutions for nonlinear fractional functional differential equation. Comput. Math. Appl. 64, 3456-3467 (2012)

26. Li, C, Luo, X, Zhou, Y: Existence of positive solutions of the boundary value problem for nonlinear fractional differential equations. Comput. Math. Appl. 59, 1363-1375 (2010)

27. Yang, J, Ma, JC, Zhao, S, Ge, Y: Fractional multi-point boundary value problem of fractional differential equations. Math. Pract. Theory 41(11), 188-194 (2011)

28. Rehman, $M$, Khan, $\mathrm{R}$, Asif, N: Three point boundary value problems for nonlinear fractional differential equations. Acta Math. Sci. 31(4), 1337-1346 (2011)

29. Cernea, A: On a multi-point boundary value problem for a fractional order differential inclusion. Arab J. Math. Sci. 19(1), 73-83 (2013)

30. Zhou, W, Chu, Y: Existence of solutions for fractional differential equations with multi-point boundary conditions Commun. Nonlinear Sci. Numer. Simul. 17, 1142-1148 (2012)

31. Jiang, W, Wang, B, Wang, Z: The existence of positive solutions for multi-point boundary value problems of fractional differential equations. Phys. Proc. 25, 958-964 (2012)

32. Zeidler, E: Nonlinear Functional Analysis and Its Applications I: Fixed-Point Theorems. Springer, Berlin (1985)

10.1186/1687-1847-2014-151

Cite this article as: Li et al.: Multi-point boundary value problems for a class of Riemann-Liouville fractional

differential equations. Advances in Difference Equations 2014, 2014:151 